\title{
Intégration européenne et échanges politiques territorialisés
} Mr Emmanuel Négrier

\section{Citer ce document / Cite this document :}

Négrier Emmanuel. Intégration européenne et échanges politiques territorialisés. In: Pôle Sud, n³, 1995. L'Europe au sud. pp. 38-54;

doi : https://doi.org/10.3406/pole.1995.909

https://www.persee.fr/doc/pole_1262-1676_1995_num_3_1_909

Fichier pdf généré le 29/03/2019 


\section{Intégration européenne et échanges politiques territorialisés}

\section{par Emmanuel Négrier}

La thèse la plus généralement soutenue à propos des relations entre construction européenne et régionalisation est celle d'une relation de cause à effet entre la première, le développement des programmes communautaires et une stimulation des régions comme acteurs de plus en plus importants dans ce processus. Cette thèse repose sur un certain nombre de paramètres, parmi lesquels : l'impact des réformes successives de la politique régionale européenne sur la mobilisation (par le bas et par le haut) des acteurs régionaux; l'émergence d'une représentation subnationale institutionnalisée au cœur même du processus politique européen (le Comité des Régions notamment) ; l'essoufflement de la capacité des États à "capturer" l'essentiel des relations centre-périphérie, dans son propre "sanctuaire" d'abord, vis-à-vis de l'espace communautaire ensuite.

Au delà de ses fondements politico-institutionnels, cette thèse se nourrit ensuite des acquis des analyses sur les nouvelles formes d'organisation économique postérieures au modèle fordiste. La ré-interrogation des relations public-privé à l'échelle locale et régionale, et notamment dans le cas de la troisième Italie, débouche sur un cadre économique et social de pertinence de la régionalisation dans les sociétés démocratiques européennes (Bagnasco, 1994). Même si les limites de cette thèse apparaissent désormais clairement, notam- ment quant à sa généralisation au delà de son territoire de naissance (Zeitlin, 1994), elle constitue encore l'ancrage majeur de cette eurogénèse des régions, par laquelle l'économique rejoint le politique.

Quel que soit par ailleurs son bien-fondé programmatique, elle se heurte, dans l'analyse des politiques publiques désormais disponible, à deux types d'obstacles : Le premier est l'hypothèse implicite que le niveau politique régional est, en pratique, systématiquement privilégié dans la définition et la mise en œuvre des politiques régionales européennes. On pourra ici objecter que si l'histoire des politiques régionales montre à l'évidence un "intérêt" à la diversification des interlocuteurs de la Commission comme espace de politiques publiques, et notamment sur le plan territorial, les pouvoirs régionaux ne sont, en tant que tels, qu'un des "partenaires valables" (Smith, Smyrl, 1995). Ils ne sont ni le seul, ni toujours le plus pertinent à l'examen de la mise en cuvre de ces politiques. D'autre part, le modèle de la "state capture" peut s'avérer plus pertinent à un second niveau d'analyse, lorsqu'on met l'accent sur les effets, contre-intuitifs, de dépendance des “régions" à l'égard des États dans le contexte des politiques régionales européennes (Anderson, 1990). Enfin, la confirmation du niveau régional comme niveau privilégié de mise en œuvre des politiques européennes peut signifier 


\section{Intégration européenne et échanges politiques territorialisés}

davantage le renforcement des services régionaux de l'État (préfecture, SGAR...) que celui des pouvoirs décentralisés : les conseils régionaux (Balme, Jouve, 1994).

Le niveau de généralité de la thèse ellemême est le second obstacle. Il s'avère en effet souvent contradictoire avec la diversité forte des régions (leur capacité institutionnelle, économique, leur taille et légitimité historique, leur capacité de médiation et de représentation des intérêts...) dont il est question. Cette approche, par trop globale, conduit à traiter, dans un même ensemble, non seulement des régions de statut constitutionnel et national différent, comme suffit à le montrer l'incongruité d'une assimilation entre le Bade-Wurtemberg et la Basse-Normandie, mais aussi des régions qui, pour être de même statut national, n'en sont pas moins très fortement contrastées quant à leur histoire politique, leur rapport à l'État (Genieys, 1994) ou encore leur capacité politique (Ritaine, 1995). La comparaison, instruite de ces limites à l'équivalence généralisée, peut néanmoins s'attacher à démontrer sur quoi reposent les plus opératoires critères.

On avancera donc l'hypothèse selon laquelle il est possible de parler des régions comme acteurs politiques de l'intégration européenne en fonction de certains critères, dont les suivants : elle peuvent offrir un relais institutionnel de communication entre intérêts publics et privés; elle peuvent mobiliser des ressources politiques et administratives de nature à les protéger des conséquences non voulues de l'intégration européenne; elles peuvent représenter des forums territoriaux de politiques publiques européennes.
Cette hypothèse suppose la prise en compte des réseaux formés ou mobilisés à l'occasion de la mise en œuvre des programmes européens. Lanalyse des réseaux est ici conçue comme le moyen d'expliciter et d'évaluer la portée de phénomènes considérés comme croissants dans l'action publique contemporaine (Kenis, Schneider, 1991) : l'interdépendance entre des acteurs plus nombreux et différenciés ; la sectorisation et la fragmentation de l'État, qui s'accompagnent de la croissance des régulations informelles sur les arène officielles de débat ou de gestion; la densité des relations public et privé; le rôle des intérêts privés organisés dans la définition de l'action publique ; l'internationalisation des contextes d'action, mettant en crise la spécificité de chaque modèle.

L'analyse des réseaux et communautés permet ici d'apprécier les transformations éventuelles induites par l'européanisation des contextes d'action régionale sur les modes d'effectuation des politiques publiques (Hassenteufel, 1995). Ces transformations ne sont pas seulement analysables en termes stratégiques. L'optique relationnelle d'une telle analyse ne serait en effet que d'une piètre originalité s'il lui suffisait d'adapter les arcanes de la sociologie des organisations à des contextes d'action contemporains (Mény, 1989 ; Lazega, 1993 ; Jouve, 1995). Pour délicate qu'elle soit à l'échelle strictement régionale, l'étude des réseaux doit également se donner pour objet le contenu des échanges, leur dimension politique notamment. D'une part, les communautés dont il est question ne sont pas simplement construites sur des dimensions strictement opérationnelles. Elles ont 


\section{Pôle Sud}

souvent une histoire de longue durée, y compris sous les apparences les plus modernistes des programmes européens. Elles sont ensuite structurées par des phénomènes de croyance, de valeurs partagées, qui permettent de les qualifier de communautés "épistémiques" (Haas, 1993). La notion de communauté épistémique permet par exemple de dépasser les analyses traditionnelles en termes de décalage entre préconisations à l'échelle européenne et dérives successives liées à la mise en œuvre. $\mathrm{Au}$ moins, elle leur donne un cadre qui échappe au jugement de valeur, plus ou moins habilement déguisé, puisqu'elle prend ces mêmes valeurs comme une donnée dépendante.

Ainsi, les différents modes de mise en œuvre des Programmes Intégrés Méditerranéens (PIM) nous renseignent-ils sur les formes très différentes de gestion politique régionale, non seulement du point de vue des contenus définis, mais aussi des formes d'accords régionaux qui divergent selon la communauté de valeurs que la région, de par son histoire, sa structuration économique, ses degrés de fragmentation politique, etc., actualise ou non. Du reste, la notion de communauté épistémique peut être également utilisée pour appréhender les degrés de cohésion politique des modèles d'action communautaire eux-mêmes. Il n'est pas sûr, contrairement là aussi aux idées toutes faites sur l'Union Européenne, que la Commission soit en mesure d'incarner un modèle d'action unique ou même cohérent, quand on l'observe de près, c'est-à-dire du point de vue des arènes bureaucratiques propres à chaque direction générale et même à chaque division (Richardson, 1994). L'Union Européenne apparaît in fine non seulement comme une sphère de gouvernance à plusieurs niveaux, comme le montrent déjà, du point de vue de l'articulation des intérêts sectoriels, certaines analyses (Fuchs, 1994 ; Négrier, 1994 ; Smith, 1995), mais aussi une sphère de discussion entre des communautés différenciées par des valeurs de référence, et orientées vers l'action.

L'observation d'une série de politiques communautaires en région LanguedocRoussillon permet de rendre opératoires certaines de ces hypothèses. La présentation que nous allons en faire, part de l'analyse de formes différenciées de réseaux de politiques publiques, selon une logique plutôt descriptive. Elle est suivie d'une interrogation plus dynamique sur les échanges politiques à l'œuvre dans les processus de négociation, de (re) définition des programmes et de mise en œuvre. Elle se conclut par un certain nombre d'enseignements, provisoires, sur la "capacité" des régions à représenter un forum des politiques publiques européennes sur leur territoire.

\section{Médiations et réseaux régionaux des politiques européennes}

Trois caractères nous suffirons ici pour donner un cadre à l'incidence des politiques européennes en LanguedocRoussillon :

- Eu égard à sa tradition viticole et agricole, et à la faiblesse historique de son appareil industriel, la région LanguedocRoussillon illustre, sans doute mieux que 


\section{Intégration européenne et échanges politiques territorialisés}

toute autre région, la ruralisation des politiques structurelles en France, qui relève notamment d'un choix politique de l'État (Alliès, Lacave, 1994). Elle est la troisième région française pour l'attribution les fonds structurels concernant le développement rural (Objectif 5b), avec 43 écus par habitant (derrière Champagne-Ardennes et Provence-Alpes-Côte d'Azur). Mais elle est également la première pour ce qui concerne l'Objectif 2 (zones en reconversion industrielle), avec 42,6 écus par habitant.

L'Union Européenne incarne en L.-R. une palette importante et diversifiée d'interventions : au delà des zones structurelles, divers Programme d'Initiative Communautaire (PIC) interviennent dans les domaines transfrontaliers (INTERREG), de la recherche et développement (SPRINT), des études liées aux parcs technologiques (STRIDE) ou autres opérations de développement en réseau (LEADER). Sa contribution est en hausse (1,5 milliards de francs pour 94-99) et représente désormais une dimension essentielle du développement régional et infra-régional. La contribution de l'Europe au Languedoc-Roussillon (en 1989-1993) équivaut à plus de la moitié des fonds que l'État lui affecte par le biais des contrats de plan Etat-Région.

- La précocité d'un mode d'accès à Bruxelles comme ressource stratégique s'explique par le fait que, parmi les rares zones françaises à préfigurer les nouvelles orientations de la politique européenne des années 1980, la Lozère a expérimenté, la première, la programmation intégrée des opérations de développement initiée par la Commission. Elle se fonde surtout sur l'expérience des Programmes Intégrés
Méditerranéens (PIM) qui ont constitué la première, et peut-être la plus intense forme de régionalisation des politiques européennes (Smyrl, 1995b). De cette précocité programmatique dérive aussi le fait que l'Europe a, très tôt, constitué un mode de légitimation territoriale et politique, pour laquelle les initiatives ont largement excédé les limites de l'institution régionale et les stratégies de son président.

- La forte fragmentation des acteurs institutionnels du développement économique, où chaque niveau peut prétendre à une parcelle de pouvoir et de légitimité ; cette fragmentation pose largement la question de l'existence et de la pertinence du niveau régional comme espace d'articulation des intérêts organisés et des différents niveaux d'action locale, comme dans bien d'autres régions françaises (Mény, 1990 ; Alliès, 1989). Elle est la traduction, pour ce qui concerne le développement économique, de lignes de clivage régionales qui tiennent tout autant des formes de représentation politique d'une économie singulière, mono-viticole (le "Midi rouge"), que d'une segmentation religieuse, géographique et industrielle du territoire régional. Ces traits fragmentaires ont été, dans une large mesure, au principe de comportements politiques et sociaux, structurellement divers, qui se sont eux-mêmes traduits par des conflits inter-institutionnels avivés par les politiques de décentralisation (Ritaine, 1991 ; CEPEL, 1987, 1989, 1994 par exemple)

Ces trois caractères languedociens offrent donc un cadre à la mise en œuvre des programmes européens. Celle-ci est d'autant plus intéressante à analyser que, 


\section{Pôle Sud}

contrairement aux politiques territoriales nationales, le niveau principal d'impulsion politique, la Commission Européenne, ne dispose pas de l'équivalent d'une administration déconcentrée. Ensuite, les principes de partenariat, d'additionnalité, de programmation, de subsidiarité, qui forment classiquement le référent de l'action communautaire, sont à la fois contraignants (formellement) et souples (dans les schémas d'acteurs locaux qu'ils autorisent). C'est donc sur des réseaux d'acteurs, au degré de cohésion variable et aux références hétérogènes, qu'elle repose. Ces réseaux ne sont pas configurés selon un schéma unique. On peut au contraire démontrer qu'ils sont au principe de formes de médiation très contrastées. Une première analyse de la mise en ouvre des programmes communautaires en Languedoc-Roussillon permet d'en distinguer au moins trois.

Un premier modèle de médiation corporatiste est assuré, dans des configurations d'acteurs différentes ou semblables, par des groupes détenant une légitimité historique sur un territoire, à partir du développement économique. L'exemple du bassin de reconversion industrielle d'Alès montre que la domination sur le réseau d'acteurs du développement économique est assurée par le corps des mines qui, à travers les directions régionales, l'École des Mines et les Charbonnages de France, a fourni sur la longue durée l'essentiel du discours légitime et des stratégies autorisées sur la zone minière d'Alès. C'est lui qui est également au cœur du passage d'une conception à une autre des politiques concernant la zone : le développement des mines, les stratégies de reconversion, le développement de l'inno- vation technologique, à travers la création d'infrastructures de développement high tech (même s'il perd un peu de son influence sur ce dernier aspect). La diversité et la cohérence des ressources (qui va jusqu'à l'ancienneté de son implication dans les politiques européennes à travers la CECA) de ce corps lui permet d'influer à la fois sur les stratégies d'acteurs et sur les recettes qui conviennent. On peut donc dire que dans ce cas là, le réseau d'acteur est dominé par : une logique de reproduction d'une communauté relativement limitée et itérative dans ses logiques de relation et d'échange (économique, prospective, stratégie...) ; la permanence de la domination d'un corps technique, sur les orientations légitimes sur la zone ; l'extrême difficulté des acteurs extérieurs à cette communauté à faire valoir des orientations alternatives, l'essentiel du travail de conversion des politiques et programmes en projet de zone étant, à l'échelle européenne, sous sa dépendance. Il reste que, dans un contexte économique marqué par une gestion "politique" du retrait quasicomplet des industries minières, la légitimité du corps dans la recherche de nouvelles recettes n'est plus aussi certaine. Malgré la reconversion de l'École des Mines à de nouveaux axes d'expertise et de professionnalisation, comme d'autres Écoles nationales supérieures (exemple: l'ENSPTT), l'émergence de politiques départementales, alésiennes, ou interurbaines peut être le signe d'une pluralité d'orientations, pour laquelle la référence à l'Europe (programmes STRIDE, SPRINT) est un élément du répertoire de légitimation. 


\section{Intégration européenne et échanges politiques territorialisés}

Un second modèle de médiation d'expert, tend moins à valoriser la représentativité (politique, corporatiste, territoriale) que les capacités à accumuler de l'expérience et du savoir-faire sur les programmes européens (l'opportunité du moment, l'écheveau des procédures, les critères de recevabilité) qui se transforment en ressources stratégiques au moment où ces informations sont appliquées à des cas de figure territoralisés. On trouve une forme de "professionnalisation" au sein des institutions (régionale, départementale, municipale), des entreprises et corps consulaires : on peut estimer en L.-R. à environ 60 personnes le nombre de ces "experts". On la trouve également, apparemment dans un second temps, chez d'authentiques professionnels qui ont institué cette médiation comme l'essentiel de leur métier: les consultants et structures de lobbying.

Le cas du programme Leader "PaysCathare", essentiellement situé dans le département de l'Aude, illustre cette seconde figure de médiation. Un projet existe au départ, porté par le Conseil Général, en coordination avec des élus consulaires, puis la DATAR. Le travail de conversion entre un projet de développement économique/touristique et les programmes européens s'effectue autour de deux personnalités que l'on peut considérer, dans cette activité, comme des professionnels. Leur expertise est d'autant plus importante que l'on se situe dans un contexte d'initiative communautaire, avec un rôle plus aléatoire de l'État (au moins dans la période 89-93 étudiée) et une sélectivité des projets plus importante (logique concurrentielle).
Mais paradoxalement, cette expertise puise ses ressources dans une longue et précoce acclimatation aux politiques locales et à la vie politique locale et européenne. Elle n'en est pas dissociable, comme dans le cas de cette professionnelle du lobbying et du montage de dossier, auparavant déléguée des droits de la femme, puis attachée parlementaire d'un député européen du Languedoc-Roussillon. Cette professionnalisation de la médiation est donc bien liée à une accumulation de ressources hétérogènes et diversifiées : réseau de guichets, réseaux politiques, implantation locale, et parfois, légitimité universitaire. Ce tout constitue, en tant que tel, un réseau (interconnaissance, partage d'informations...) qui associe les différents acteurs appartenant à des institutions diverses, et dont les relations nourrissent des partenariats locaux. L'activité de médiation ne se limite pas, dans ce cas comme dans celle des "médiateurs" de P. Muller (Muller, 1991), à mettre en relation des acteurs appartenant à différentes sphères et échelles d'action. Elle se construit et se légitime au moins autant dans les opérations de traduction et d'adaptation réciproque des contenus des projets et programmes, c'est-à-dire aussi dans la mise en cohérence de systèmes hétérogènes de représentation.

Un troisième modèle de médiation politico-institutionnelle par les réseaux d'action économique locale se distingue des deux premiers. Dans ce cas, la définition des objectifs généraux s'avère insuffisante pour expliquer le cadre concret de la mise en œuvre. Un certain nombre de facteurs lourds expliquent les profils des réseaux : l'identification des contreparties financières, 


\section{Pôle Sud}

qui impliquent généralement un poids significatif dans ceux-ci de l'organisation qui en détient la plus grande charge ; la responsabilité, vis-à-vis des institutions de financement des programmes, de la validité des critères retenus : elle explique le rôle du SGAR (Secrétariat Général aux Affaires Régionales, près la Préfecture de Région) dans le déroulement des procédures.

Mais ces facteurs ne permettent pas d'expliquer les médiations, jusqu'aux projets retenus, des programmes : leur diffusion, leur interprétation dans les contextes locaux d'action, leur sélection à la fin du processus. Pour les expliquer, il faut analyser les réseaux concrets de développement local, et notamment l'inscription sur le territoire de médiateurs du développement, autour des conseils généraux par exemple. Le cas de la mise en ouvre du PDZR (Plan de Développement des Zones Rurales) dans l'Hérault est typique de ce modèle. Sa conception originelle confiait à la région un rôle central. L'absence de mise sur pied d'une cellule spécifique à l'échelon régional pour coordonner l'ensemble des programmes PDZR de la région, de même que le refus, par l'État, de consacrer un rôle d'articulation au Conseil Régional (Blanc, 1995) sont deux explications, de nature négative, souvent avancées. On émettra également l'hypothèse positive que les réseaux de développement local, à l'intérieur de la région, se structurent à un tout autre niveau. La mise en réseau des chartes intercommunales et les acteurs locaux, publics et privés, appuyée sur la contrepartie financière départementale, constitue une structure déterminante à cet égard. À l'intérieur de celle-ci, un rôle majeur peut être joué par l'acteur qui parvient à assurer la convergence entre les programmes (dans la période 1989-93, il s'agit de programmes nouveaux, peu connus, dont les enjeux ne sont pas socialisés a priori) et les acteurs intermédiaires (représentants consulaires, élus locaux, destinataires finaux). Cette mise en cohérence n'est pas exempte de conflits, de tentatives de récupération, de délégitimation tentées. À l'origine du PDZR, les consulaires tentent ainsi d'exclure les chartes, avec lesquelles ils sont pourtant en relation sur le développement local, du pilotage du PDZR. On le voit très clairement dans l'analyse des différents canaux de remontée de l'information sur les projets individuels, où la domination du département est très nette. Il est d'autant plus intéressant de travailler cet exemple que, dans le cadre du même programme, on voit combien l'absence de préstructuration d'un réseau de développement local dans l'Aude conduit à la gestion par la préfecture de l'intégralité du dossier et à la tentative, sur le tard, de structurer un réseau d'animateurs locaux apte à conduire, pour l'avenir, de telles politiques.

Ces trois types de médiation sont certes de simple modes dominants dans les cas étudiés. Ils ne sont pas exclusifs de la présence des autres, mais dessinent cependant un paysage de réseaux très contrasté d'une politique à l'autre. Ils nous renseignent ensuite sur des tendances communes :

- ils sont en général ancrés dans une histoir, qui mêle les profils individuels, les appartenances politiques et des logiques de représentation et d'action territorialisées (par un groupe, un parti, une institution); 


\section{Intégration européenne et échanges politiques territorialisés}

- ils dépassent nettement les limites traditionnelles de la "mise en œuvre", lorsqu'ils concourent à la mise en cohérence, intellectuelle autant qu'opérationnelle, de politiques territorialisées ;

- ils permettent d'affirmer que les "réseaux locaux de l'Europe" sont pluriels, qu'ils entrent en contact de manière également diverse avec les institutions officiellement en charge de programmes : La région, la DATAR, les préfets et le SGAR. Autrement dit, on peut analyser la mise en œuvre territoriale des programmes communautaires comme révélatrice d'une articulation, problématique, entre des réseaux de projets (issue neworks), fragmentés, et une communauté formelle de politiques publiques européennes. Cette dernière, pour incarner entre ses membres des relations denses et itératives, n'en est pas moins un élément seulement de l'écheveau, à la cohérence incertaine.

\section{Référentiel européen et échanges politiques}

Avec la mise en évidence de réseaux de mise en œuvre à la fois divers et fragmentés, nous en étions restés à une vision relativement statique de l'analyse des politiques publiques. Nous voudrions ici dépasser cette étape nécessaire, en nous interrogeant sur le contenu dynamique des échanges qui découlent de et construisent ces réseaux. Deux questions se posent essentiellement ici : ces réseaux construisent-ils au plan régional une définition homogène de la réalité sociale et des principes d'action européenne sur laquelle se fonderait en conséquence un référentiel d'action régionale communautaire? Les échanges auxquels donnent lieu ces réseaux de politique peuvent-ils être considérés comme des échanges politiques eux-mêmes, et avec quelles conséquences pour l'analyse des régions comme acteurs politiques européens?

\section{Référentiel européen et mise en cohérence à plusieurs niveaux}

Quant à la première question, distinguons deux points importants. Tout d'abord, l'observation des contextes d'action concrets montre que l'ensemble des principes d'action communautaire (additionnalité, programmation, concentration, partenariat), de même que la notion de subsidiarité constituent en tant que tels un référentiel, au sens que $B$. Jobert, à la suite de Kuhn, donne à ce terme : "les solutions concrètes qui, réemployées comme modèle ou comme exemple, peuvent remplacer les règles explicites (...) et apparaissent comme autant de normes permettant de réguler les interactions" (Jobert, 1995). La première particularité de ces normes est ici qu'elles participent à la fois des domaines explicite et implicite. Autrement dit, le principe de partenariat n'est pas simplement un prérequis institutionnel pour l'ouverture d'un guichet européen, mais aussi une nouvelle vision du monde rapportée à l'action publique locale. On peut inscrire, dans une telle nouvelle représentation, les dimensions de politique symbolique attachées à l'Europe dans les discours des élus régionaux, mais aussi des stratégies, à la charnière entre cette dernière et l'organisation de nouveaux espaces de pertinence 


\section{Pôle Sud}

publique que sont les divers réseaux de villes, de régions... qui donnent une réalité politique à ce principe de partenariat ( $\mathrm{F}$. Morata, dans ce même numéro, en analyse l'une des illustrations). On pourrait faire la même remarque pour les notions de subsidiarité et d'additionnalité qui, au delà de leur prétention à simplifier les cadres d'action, construisent des représentations intellectuelles en regard des relations entre acteurs, publics et privés. La seconde particularité de ces normes, au vu, précisément, de leur statut ambivalent, est qu'elles autorisent des pratiques extrêmement variables d'un contexte à l'autre. Loin de pouvoir incarner une armature très précise, elles constituent une base seulement pour les échanges politiques, à l'échelle régionale par exemple. C'est donc dans le cadre flou de ces normes que se construisent les alternatives politiques, tant au plan territorial qu'au sein de la Commission elle-même. Il n'est qu'à mentionner les conflits qui opposèrent, aux deux niveaux, les différents protagonistes des Programmes Intégrés Méditerranéens pour se persuader de l'espace ouvert d'interaction et d'échange aux deux bouts de la chaîne d'action publique. Les critiques faites à l'utilisation de l'analyse des réseaux dans le domaine des politiques communautaires reposent précisément sur cette conflictualité, qui n'est que le reflet de situations où prévaut une juxtaposition de décisions individuelles sur l'organisation de réels secteurs ou soussecteurs (Kassim, 1994). Si l'on ne doit pas forcément suivre un tel argument, notamment parce qu'il sous-estime la stabilité relative de certaines politiques européennes et des réseaux qui les structurent (politique agricole, politique de télécommunication et... politique régionale notamment), on doit au moins considérer cette fragmentation comme un acquis à l'intérieur d'un domaine particulier comme pour l'ensemble du champ des politiques européennes (Peterson, 1995).

Il devient par conséquent difficile, et c'est là le second point important, de maintenir, sauf pour des préoccupations directement opérationnelles, un cadre intellectuel de mesure de la distance entre définition et mise en œuvre des politiques. Que celle-ci verse dans la dénonciation d'éventuelles pratiques corruptives (D'Aubert, 1994), ou qu'il s'agisse de mesurer telle ou telle distance cognitive entre amont et aval, elle bute sur la double incertitude dans l'élaboration des principes et leur (re) construction ou traduction dans des contextes élargis. La sanction d'écarts vis-à-vis d'une norme requiert au préalable une assez grande certitude sur les contours de cette norme. Admettons cette condition remplie, au niveau assez global de l'ensemble des recettes d'action communautaires, c'est-àdire en relativisant les conflits qui participent de leur construction et de leur mise en cohérence. Il faut ensuite considérer que ce dernier processus s'achève avant même que de concerner les acteurs territoriaux. Or, tout montre que derrière cette image simple de mise en œuvre, bien des phénomènes viennent contredire l'apparente évidence. Elle néglige d'abord le rôle du Comité des Régions comme institutionnalisation de cette participation à un premier niveau d'influence. Surtout, elle fait l'impasse sur l'effet des traductions à l'œuvre dans les réseaux (cf. supra), qui peuvent être analysés 


\section{Intégration européenne et échanges politiques territorialisés}

comme d'authentiques définitions des normes d'action, et non leur seule application, déformée ou non. Elle passe également sous silence le processus de légitimation, dans les deux sens, dont cette "mise en cuvre" est indissociable, et qui participe de l'échange politique.

Ainsi, les milieux de médiation des programmes qui sont liés à des histoires et des institutions, ne font pas qu'en reproduire les simples logiques d'action ni même les clivages figés. Il faut au contraire penser l'articulation entre d'une part les réseaux diffuseurs de références, $d$ ' "acculturation européenne", et d'autre part les réseaux territoriaux qui intègrent les exécutifs locaux, les groupes d'intérêts et les acteurs micro-territoriaux. Deux types de solidarité contraires sont en tension : la solidarité avec l'institution où l'essentiel de la légitimité se construit et la solidarité avec le groupe "européiste", même informel et peu structuré, où l'information stratégique s'échange. C'est au croisement de cette tension que s'invente le référentiel local-européen.

La mise en œuvre de programmes européens actualise et accompagne parfois la création de nouvelles politiques. Dans l'Aude, la création d'un tissu d'acteurs du développement local repose en partie sur les leçons tirées de l'échec de la première phase du PDZR (faible implication du conseil général, notamment vis-à-vis de l'Hérault) et l'importante mobilisation dans le cadre de l'opération Leader "PaysCathare" : ces nouvelles politiques articulent elles-aussi des dimensions exogènes (les principes d'action communautaires) et des dimensions endogènes : la légitimation d'acteurs du développement local s'opère dans un contexte de renouvellement du personnel politique, notamment à l'intérieur du PS et du conseil général audois.

La production d'un référentiel d'action régionale communautaire est donc liée à une construction à plusieurs niveaux des recettes et des réseaux qui les portent. Par $\tan t$, elle doit assurer, pour ce qui nous concerne, un double rapport à la globalité : celui de son articulation avec un hypothétique référentiel européen, dont l' "économie sociale de marché" fournit pour certains la clef, et dont on peut penser qu'il comporte également une dimension politique d'intégration à plusieurs niveaux (Schmitter, 1992) ; celui de sa mise en cohérence avec les représentations et modes d'action publique territoriale. En ce sens, il devient important d'identifier, outre le rôle que joue l'autorité régionale dans ces politiques, celui qu'elle est amenée à assumer (ou non) en termes de politiques publiques et, plus largement, d'échange politique.

\section{Un échange politique territorialisé}

Dans la logique de ce qui précède, la nature même des échanges entre différents niveaux devient centrale. Elle met, en effet, non seulement en question les types d'interaction entre acteurs, mais aussi la création et la routinisation des recettes d'une politique territoriale, au sens où la définit $R$. Balme (Balme, Ritaine, 1994). De ce point de vue, le modèle de l'échange politique territorialisé de L. Parri (Parri, 1990) fournit un cadre partiellement satisfaisant. En s'intéressant aux relations de négociation institutionnalisées entre gouvernement et collectivités locales, où s'échangent 


\section{Pôle Sud}

inflexion d'une politique territoriale contre consensus élargi sur sa mise en œuvre, il n'apporte pas d'innovation analytique majeure au modèle classique de la régulation croisée (Grémion, 1976). Ce dernier demeure d'ailleurs heuristique, lorsqu'il s'agit de décrire les relations qui se nouent entre les niveaux de formulation des projets, de négociation des orientations légitimes, et de sanction financière et réglementaire. Le rôle du SGAR dans ces procédures constitue une belle illustration de cette capacité à échanger un consentement entre de multiples acteurs territoriaux contre l'assurance (que seul le SGAR peut prendre en fonction de son statut de responsable juridique final de la conformité des opérations) que d'éventuelles dérives quant aux règles établies (additionnalité, partenariat, etc.) ne seront pas sanctionnées. La capacité d'influence de l'État (déconcentré) sur les dispositifs communautaires passe fréquemment par la mise en évidence de telles "recettes" de la régulation croisée.

La notion d'échange politique territorialisé permet pourtant, au delà du cadre strict des interactions "stratégiques", de rendre compte des processus de négociation entre acteurs. Par exemple, le partenariat dans le PDZR Aude-Hérault (1989-1993) entre les représentants consulaires et les animateurs de chartes pose un problème crucial : la lutte de position sur la notion de développement local : qu'est-ce que le développement local ? Est-il du ressort de la collectivité locale ou de ses structures affiliées? Pour les premiers, le pouvoir local doit rester cantonné à une politique d'aménagement en général : "ce ne sont pas des spécialistes (des politiques)", répond le représen- tant consulaire. Pourtant, la tentative de délégitimation des partenaires départementaux (conseil, chartes, etc.) tourne court. Est-ce du ressort des corps consulaires? Pour le conseil général, ils doivent limiter leur action aux prestations de services aux ressortissants. La thèse est également impraticable. Par le jeu des cofinancements (les contreparties), et par sa mobilisation sur le long terme dans ce domaine, le département est incontournable. La règle du partenariat impose parallèlement les représentants consulaires. Ce conflit se règle par une répartition des rôles de pilotes entre deux programmes : les corps consulaires se verront plus ou moins implicitement accorder le leadership sur le programme Leader Montagne-Hérault et le département assurera la responsabilité majeure du PDZR. Dans cet exemple, comme dans d'autres cas, notamment dans le bassin minier d'Alès, la pluralité des opérations communautaires (Objectif 2, programmes STRIDE, LEADER, INTERREG, etc.) permet d'une part un accès élargi à plusieurs types d'acteurs au label et à la manne européenne. Elle permet par ailleurs de fournir des voies de résolution, symboliques et matérielles, des négociations en cours.

En quoi le processus qui vient d'être décrit est-il justiciable d'une analyse en termes d'échange politique ? Pour au moins trois raisons : la question posée est celle de la légitimité et de l'influence déterminante que deux types d'acteurs en relation vont détenir sur des politiques publiques, et du traitement des rapports de force auxquels cette question renvoie; la résolution du conflit débouche sur une légitimation réciproque, chacun conservant un statut de 


\section{Intégration européenne et échanges politiques territorialisés}

partenaire pour l'autre selon une division implicite du travail politique. L'échange ponctuel s'insère dans une dimension plus large de relations, itératives, qui sont autant faites de coopération que de conflits, de clivages institutionnels que d'interpénétrations.

La conquête d'une position de leader sur un programme n'est pas sans lien avec les stratégies électorales respectives, auprès des ressortissants ou des électeurs. Dans cet exemple, elle est une des ressources de légitimation d'un représentant consulaire dans la compétition politique sur un siège de conseiller général. Ainsi, c'est précisément la dépendance de cet échange circonscrit à l'égard d'un espace politique plus large qui en définit le plus sûrement la nature d'échange politique territorialisé. Ce faisant, celui-ci s'affranchit d'une stricte détermination stratégique, ou fonctionnelle, puisqu'il puise dans des registres de légitimation, réciproque, sur un espace politique élargi, mais aussi dans une histoire de ces relations qui relativise l'effet d'innovation radicale des issue networks. Faut-il pour autant en conclure que, dans l'ensemble des réseaux de relations, l'échange politique confirmerait l'hypothèse néo-corporatiste tandis que le marchandage (bargaining) appuierait au contraire la thèse pluraliste (Crouch, 1990) ? Cette discussion excède les limites de notre présentation, mais la simple variété des modes de médiation ( $c f .1^{\text {tre }}$ partie), qui, intégrant ce type d'échange, tiennent pourtant les uns du premier, les autres du second courant, incite à douter d'une telle simplification.

Conformément au modèle de L. Parri, l'échange politique territorialisé s'étend à d'autres catégories d'acteurs et à d'autres espaces que le simple département dont il vient d'être question. Cette "chaîne" d'échanges, présente au niveau du SGAR comme on l'a déjà mentionné, se reproduit jusqu'à la Commission Européenne. On peut ainsi penser que les fonctionnaires font parfois prévaloir la recherche d'interlocuteurs valables (par la fiabilité de leurs réseaux, l'exemplarité formelle de leurs projets, la visibilité de leurs opérations) sur la conformité des normes appliquées. Là aussi, l'échange politique permet de traduire des effets de légitimation, comme par exemple : la possibilité de contredire la réputation de bureaucrate incompréhensif, ou le discours itératif sur le déficit démocratique ou le défaut de proximité de Bruxelles à l'égard du terrain ; le surcroît de légitimité que donne, à ceux des acteurs "de terrain" de la Commission, la possibilité de se prévaloir d'interlocuteurs territoriaux à la fois "complices" et extérieur à l' "étau" des Étatsmembres; la capacité de représenter, en tant que personnage notable, la Commission au niveau territorial.

$\mathrm{Au}$ total, cet échange politique territorialisé puise certaines de ses caractéristiques dans le modèle de la régulation croisée. Mais il s'en distingue sur des points essentiels : une pluralité d'acteurs d'horizons distincts participent à ce processus d'échange en chaîne; l'incertitude même des règles qui président au "sommet" européen interdit de penser simplement en termes d'ajustements locaux vis-à-vis d'un bloc hiérarchique et sûr, comme cela était le cas dans le modèle du CSO ; si la cohérence référentielle européenne dépend de processus de traduction locale, intégrant la singularité 


\section{Pôle Sud}

des espaces, des histoires et des systèmes politiques, il s'en suit que les échanges politiques territorialisés peuvent largement différer d'une région à l'autre. Il faut par conséquent ne pas faire disparaître derrière le principe de l'échange la spécificité du lieu où il se concrétise. Il n'est ainsi pas évident que l'on puisse parler partout, à l'occasion des politiques européennes, d'échange politique régionalisé, comme on a pu le faire avec la régulation croisée.

\section{Un échange politique régionalisé?}

Nul doute que les régions sont aujourd'hui parvenues à s'imposer comme acteurs des politiques européennes. Mais derrière ce constat général, comment peuton qualifier la nature de leur intervention, et ce notamment vis-à-vis des différents niveaux publics territoriaux et des intérêts privés organisés ? On peut avoir la quasicertitude qu'il existe de substantielles différences sur ce point entre les régions françaises elles-mêmes. Ainsi, M. Smyrl (1995a), comparant la mise en œuvre des fonds structurels en Bretagne et en Provence-Alpes-Côte d'Azur, met en évidence plusieurs divergences intéressantes, parmi lesquelles : la logique de consensus et de socialisation des informations entre niveaux, qui prévaut à l'échelle régionale en Bretagne, s'oppose à la continuité de rapports de force et à l'autonomisation des réseaux d'information en PACA; les accommodements vis-à-vis des normes sont plus faciles et fréquents en Bretagne, alors qu'en PACA prévaut un comportement tatillon (sur les plans réglementaire et financier) de la part de la préfecture de région; l'influence de la préfecture de région n'est jamais aussi importante dans le dispositif européen que lorsque les acteurs régionaux sont fragmentés, en conflit et, à l'inverse, est contrainte de compter avec l'autorité politique régionale lorsque, comme en Bretagne prévaut un relatif consensus.

On peut en déduire que, d'une part, les différences sont fortes d'une région à l'autre et que, d'autre part, ces différences sont liées à la capacité ou non de la région à incarner un forum des politiques européennes la concernant. On pourra parler de forum régional des politiques européennes si l'on peut constater : sa capacité à agréger les intérêts selon une logique à la fois verticale (entre différents niveaux) et horizontale de représentation des intérêts privés et publics ; sa capacité à construire un compromis territorialisé qui repose sur la régulation des interactions entre différents réseaux (fonctionnaires européens et nationaux, déconcentrés ou non, experts, agents de développement, scientifiques, représentants professionnels, corps techniques, etc.).

Cela implique que le conseil régional représente, à travers ses politiques et son propre statut, plus qu'une arène, c'est-à-dire un simple espace de négociation des politiques, en concurrence avec d'autres arènes (Jobert, 1995). La notion de forum régional suppose qu'il soit le lieu privilégié des débats de politique européenne, où se construisent les représentations de la réalité économique et sociale, s'établissent les compromis régionaux. Autrement dit, il s'agit de chercher à identifier un échange politique régionalisé qui se détache significativement de la notion de marchandage par son rapport territorial aux représentations, à 


\section{Intégration européenne et échanges politiques territorialisés}

la légitimation. Pour reprendre nos deux exemples, il semble clair que la Bretagne incarne assez bien un tel modèle d'échange politique régionalisé mais que, par contre, la région PACA ne peut y correspondre.

La région Languedoc-Roussillon est, de ce point de vue, finalement assez comparable à la région PACA. Quelques constats l'illustrent :

- alors même qu'il s'agit d'une région pionnière vis-à-vis de l'Europe, l'indécision quant à la gestion interne des questions européennes (une Mission Europe généraliste, une déconcentration par services) au sein du conseil régional s'est poursuivie jusqu'en 1994 ;

- la plupart de nos interlocuteurs soulignent le doute sur une capacité de coordination régionale des programmes sectoriels européens (tant en matière rurale qu'en matière de recherche et développement ou de reconversion industrielle). Si cela est dû, pour le rural, à la faiblesse comparative de son maillage territorial, une telle raison n'est pas propre au conseil régional languedocien ;

- le milieu des experts européens de différents niveaux, régional et sub-régional, public et privé, demeure davantage clivé par des logiques d'appartenance institutionnelle qu'unifié dans une communauté "épistémique" ou stratégique. L'intensité des relations entre eux demeure faible.

- sur la période 1989-1993, la région n'a pu ni imposer un programme opérationnel unique, ni la cohérence des zonages européens $(2,5 \mathrm{~b} \ldots)$ avec ses propres circonscription d'action territoriale.

- lors du renouvellement des zones, la région a de même été dans l'incapacité de faire avaliser des choix, notamment la restriction au Gard des zones Objectif 2 de reconversion industrielle, qu'elle privilégiait de manière constante.

L'activité européenne du conseil régional, limitée dans son rôle de porte-voix des acteurs publics et privés régionaux (par exemple sur les zonages), et dans sa capacité d'agréger les intérêts sur cet ensemble de politique, semble privilégier une dimension de "représentation externe" de la région : "militantisme" précoce au sein des organismes régionaux européens (ARE puis Comité des Régions), mise en exergue de l'Euro-Région, prise de parole sur les débats concernant les grandes infrastructures (TGV franco-espagnol), mise sur pied d'une agence méditerranéenne de l'environnement, etc. Cette recherche de légitimation "par-le-haut" semble être un indice supplémentaire de ses limites à l'échelle régionale pure.

Ce déficit est certes dû à des critères généraux souvent mentionnés et qui ont leur importance : réticence des instances étatiques à la régionalisation, incohérence du système électoral, capacité budgétaire et législative limitée ou nulle, etc. Mais le fait même qu'il s'applique à toutes les régions et que seules certaines d'entre elles parviennent à le dépasser ne le rend pas précisément déterminant. En sens inverse, les évolutions actuelles (intégration plus poussée des financements communautaires dans les contrats de plan ÉtatRégion, unification régionale des documents des programmation, etc.) se traduiront vraisemblablement par des effets contrastés. 


\section{Pôle Sud}

La capacité régionale à "capturer" l'échange politique, à incarner un forum des politiques européennes, est liée à la nature des médiations qui se construisent à leur occasion, ainsi qu'à un ensemble de phénomènes qui relèvent davantage des caractéristiques nationales. De ce point de vue, la diversité des médiations, la fragmentation des réseaux et la concurrence entre niveaux (dont la région ne semble être qu'une des arènes) de gouvernance apporte, pour le Languedoc-Roussillon comme pour d'autres régions françaises, un démenti aux thèses les plus audacieuses sur la régionalisation comme processus intrinsèquement lié à la construction européenne. À côté des hypothèses prospectives, qui tendent à réserver ce pronostic pour l'avenir des politiques communautaires, et voir dans quelque innovation future un renforcement du niveau régional (en général) ou de l'autorité politique régionale comme espace pertinent de leur management public, il nous paraîtrait important de nous pencher sur les voies plus profondes d'explication des phénomènes analysés.

De ce point de vue, les perspectives ouvertes, à titre d'exemple, par R. D. Putnam (1993), nous semblent stimulantes. Comme on pourra le lire dans la revue des revues italiennes de ce volume, l'auteur a fait l'objet de critiques très vives, et souvent à juste titre, dans la communauté scientifique italienne. C'est que, au delà des doutes sur le mode de traitement des très riches données accumulées par cette équipe de chercheurs, demeure un grand scepticisme quant à l'utilisation qui est faite du différentiel de civisme entre régions. Quelques chercheurs se sont d'ailleurs d'ores et déjà essayés à une application, en France et en Belgique notamment (De Rynck, 1995 ; Smyrl, 1995a) de ces variables, avec des difficultés majeures. Nous n'entrerons pas ici dans ce débat, sauf pour signaler rapidement que, si Putnam ne pose pas les bonnes questions, et n'apporte donc pas les bonnes réponses, il pose en revanche un problème qui mérite d'être abordé : celui des racines historiques et culturelles des modes d'action publique territoriale les plus contemporains.

C'est qu'en effet l'analyse toujours plus raffinée des réseaux, des références d'action et des modes de médiation pose le problème, classique au demeurant, des limites d'une analyse synchronique. De là, il découle que si nous sommes en mesure de construire toujours plus de grille d'analyse des convergences et divergences de structuration, intellectuelle et pratique, des politiques territoriales, nous sommes le plus souvent dépourvus quant à leur explication, à la relation qu'elles entretiennent avec la société, sur le long terme. C'est ce que nous avons esquissé en mettant en évidence le rapport entre fragmentation des réseaux régionaux et histoire des clivages sociaux, politiques et culturels en Languedoc-Roussillon. L'esquisse n'est pas toujours plus belle que le tableau, qui reste à travailler. 


\section{Intégration européenne et échanges politiques territorialisés}

\section{Notes}

1. Cette analyse participe d'un programme de recherche 95-96 mené en collaboration avec le MZES Université de Mannheim (Pr. Kohler-Koch, J. Grote, M. Knodt, F. Larat, S. Umberti), l'Université de Cardiff (Pr. Laughlin, J. Mathias), l'Université autonome de Barcelone (Pr. Morata, N. Gomez-Mataran, J. Etherington), le laboratoire Rives de l'ENTPE (B. Jouve, A. Smith), dont l'objet porte précisément sur l'analyse comparée des régions comme acteurs de l'intégration européenne. Une série de publications ultérieures permettront d'en présenter les résultats comparatifs, tant quantitatifs que qualitatifs. Pour le CEPEL, l'équipe comprend M. Lacave, W. Genieys, O. Dedieu, P. Bessuges, C. Cote, F. Babinet et L. Mole, sous la responsabilité de l'auteur de ces lignes.

2. $42 \%$ des canaux d'information s'avèrent être organisationnellement liés au Conseil Général de l'Hérault : le Conseil lui-même, sa cellule Europe, le Comité Départemental du Tourisme, les Chartes (à elles seules près de la moitié), les syndicats mixtes et SEM départementales. La Région ne représente ici que $2 \%$, les Chambres consulaires $9 \%$, les mairies $11 \%$, les associations et groupes professionnels $13 \%$ et la Direction Départementale de l'Agriculture $9 \%$ (ces trois derniers, au moins, étant très directement liés eux-mêmes au Conseil Général sur ce plan).

\section{Références}

Alliès P., "Territoire régional et représentation des intérêts", Politix, n 6-7, 1989.

Alliès $P$., Lacave M., "L'impact des cofinancements communautaires sur l'aménagement du territoire", in DATAR-Crédit Local de France, Finances locales et aménagement du territoire, Paris, 1994.

Anderson J., "Skeptical reflections on a Europe of Regions : Britany, Germany and the ERDF", Journal of Public Policy, vol.10, n4, 1990.

Bagnasco A., Sabel C.F., PME et développement économique en Europe, Paris, La Découverte, 1994.

Balme R., Ritaine E., Le territoire pour politiques, Paris, L'Harmattan, 1994.

Balme R., Jouve B., "Building the Regional State : French Territorial Organization and the Implementation of the Structural Funds", in Hooghe, Wright, E.U. Cohesion Policy and National Networks, Oxford, Oxford University Press, 1994.

Blanc J., "Les régions dans l'Europe", in OIP L'identité des régions, Toulouse Privat, 1995.

CEPEL-Revue Amiras-Repères, Midi rouge, Midi bouge, $n^{\circ}$ 9, 1987.

CEPEL, "Décentralisation et nouveaux services de communication", E. Négrier (dir.) Rapport pour le Plan urbain, Montpellier, 1989.

CEPEL, "Réseaux de villes et nouveaux services de communication", R. Lauraire (dir.), Rapport pour le Plan Urbain, 1994.

Crouch C., "Generalized Political Exchange in Industrial Relations in Europe During the Twentieth Century", in Marin B. (ed.), Governance and Generalized Exchange. Self Organizing Policy Networks in Action, Frankfurt, Campus Verlag, 1990.

D'Aubert F., Main basse sur l'Europe : enquête sur les dérives de Bruxelles, Paris, Plon 1994.

De Rynck S., "The Policy Performance of the Belgian Regions. A First Exploration of a Possible Explanation", papier présenté au colloque Les régions en Europe, CERI-CRAP, Rennes, octobre 1995.

Fuchs G., "Policy-Making in a System of Multi-Level Governance : the Commission of the European Community and the Restructuring of the Telecommunication Sector", Journal of European Public Policy, 1 (2), Automne 1994.

Genieys W., Les élites face à l'État. L'institutionnalisation des élites périphériques espagnoles, Paris, L'Harmattan, 1996 (à paraître). 


\section{Intégration européenne et échanges politiques territorialisés}

Grémion P. : Le pouvoir périphérique. Bureaucrates et notables dans le système politico-administratif français, Seuil, Paris, 1976.

Haas P. M., "Epistemic Communities and the Dynamics of International Environmental Co-operation", in Ritteberger V. (ed.), Regime Theory and International Relations, Oxford, Clarendon Press, 1993.

Hassenteufel P., "Do policy networks matter ? Lifting descriptif et analyse de l'Etat en interaction", in Le Galès P., Thatcher M. (dirs.), Les réseaux de l'action publique. Débat sur les policy networks, Paris, L'Harmattan, 1995.

Jobert B., "Rhétorique politique, controverses scientifiques et construction des normes institutionnelles : esquisse d'un parcours de recherche", in Faure A., Pollet G., Warin Ph. (dirs), La construction du sens dans les politiques publiques, Paris, L'Harmattan, 1995.

Jouve B., "France : From a Regional State to Regional Governance ?", in Keating M., Laughlin J., The Political Economy of Regionalism, Londres, Frank Cass, 1995.

Kassim H., "Policy Networks, Neworks and European Union Policy-Making : a Skeptical View", West European Politics, Vol 17, N 4, 1994, pp.15-27.

Kenis P., Schneider V., "Policy Networks and Policy Analysis : Scrutinizing a New Analytical Toolbox", in Marin B., Mayntz R. (eds.), Policy Networks : Empirical Evidence and Theoretical Considerations, Frankfurt, Campus Verlag, 1991.

Lazega E., "Analyse de réseaux et sociologie des organisations", Revue française de Sociologie, 1993.

Mény Y., "Formation et transformation des policy communities : l'exemple français", in Etudes pour Georges Lavau, Paris, PFNSP, 1989.

Mény Y., "Regioni et rappresentanza degli interessi. Il caso francese", Stato e mercato, $\mathrm{n}^{\circ}$ 29, 1990.

Muller P., Politiques publiques, Paris, Que sais-je ? PUF, 1991.

Négrier E., Rabaté F., Rallet A., "L'aménagement territorial des technologies de l'information et de la communication. Stratégies et prospectives d'acteurs. Dynamiques locales, régionales et communautaires", Rapport pour la DATAR, septembre 1994.

Parri L., "Territorial Politics and Political Exchange : American Federalism and French Unitarianism Reconsidered" in Marin B. (ed.), Governance and Generalized Exchange. Self Organizing Policy Networks in Action, Frankfurt, Campus Verlag, 1990.

Peterson J., "Policy Networks and European Union Policy-Making : A Reply to Kassim", West European Politics, vol. 18, $\mathrm{n}^{\circ} 2,1995$.

Putnam R.D., Leonardi R., Nanetti R.Y., Making Democracy Work. Civic Traditions in Modern Italy, Princeton, Princeton University Press, 1993.

Richardson J., "Approches de la décision politique nationale et européenne fondées sur l'acteur : communautés politiques, réseaux par questions et communautés épistémiques", in P. Le Galès, M.Thatcher (dirs.), op. cit.

Ritaine E., "Changement social et pratiques développementalistes en Languedoc-Roussillon", Rapport pour le MRT, CERVL, Bordeaux, 1991.

Ritaine E., "La capacité politique des régions en Europe du Sud : parcours réflexif", Papier présenté au colloque Les régions en Europe, op. cit.

Schmitter P.C., "Representation and the Future Euro-Polity", Nomos Verlagsgesellschaft, n 3, 1992.

Smith A., L'intégration communautaire face au territoire, Thèse science politique, IEP de Grenoble, 1995.

Smith A., Smyrl M., "À la recherche d'interlocuteurs", Science de la société, n 34, février 1995.

Smyrl M., "From Regional Policy to European Networks : Interregional Divergence in Implementation of EC Regional Policy in France", Papier présenté à European University Institute Working, Juin 1995.

Smyrl M., "European Programs - National Structures - Regional Outcomes : Implementing the Integrated Mediterranean Programs", papier pérésenté à ECSA international conference, Charleston SC, mai 95.

Zeitlin J., "Pourquoi n'existe-t-il pas de districts industriels au Royaume-Uni ?", in Bagnasco, Sabel, op. cit. 